\title{
Szenario-Planung als Instrument einer „klimawandelangepassten“ Stadt- und Regionalplanung - Bausteine der zukünftigen Flächenentwicklung und Szenarienkonstruktion im Stadt-Umland-Raum Rostock
}

\author{
Maria Hagemeier-Klose • Meike Albers • \\ Michael Richter $\cdot$ Sonja Deppisch \\ Eingegangen: 17. August 2012 / Angenommen: 31. Juli 2013 / Online publiziert: 20. September 2013 \\ (C) Springer-Verlag Berlin Heidelberg 2013
}

\begin{abstract}
Zusammenfassung Der Klimawandel und seine Auswirkungen stellen die Raumplanung vor neue Herausforderungen, die trotz der damit verbundenen Komplexität und Unsicherheit eine nachhaltige zukunftsfähige Entwicklung gewährleisten soll. Diese Herausforderungen waren Gegenstand eines Szenario-Planungs-Prozesses für den StadtUmland-Raum Rostock, in dessen Rahmen Strategien und Ziele für die Anpassung an die Folgen des Klimawandels erarbeitet wurden. Der Prozess bezog dabei intensiv zentrale lokale und regionale Akteure ein. Aufbauend auf einer Analyse von relevanten Einflussfaktoren der zukünftigen Flächenentwicklung aus den Bereichen soziale/gesellschaftliche Aspekte, ökologische Aspekte, ökonomische und politische Aspekte sowie von möglichen Klimaänderungen wurden Szenarien erstellt. Diese verknüpfen unterschiedlich starke Auswirkungen des Klimawandels mit unterschiedlichen gesellschaftlichen und ökologischen Entwicklungen zu Szenarien der zukünftigen Flächenentwicklung des Stadt-Umland-Raums. Diese Szenarien ermöglichen der Stadt- und Regionalplanung die Berücksichtigung
\end{abstract}

\footnotetext{
M. Richter $(\varangle) \cdot$ M. Albers · Dr. M. Hagemeier-Klose

Dr.-Ing. S. Deppisch

Fachgebiet Stadtplanung und Regionalentwicklung,

HafenCity Universität Hamburg, Winterhuder Weg 29,

22085 Hamburg, Deutschland

E-Mail: michael.richter@hcu-hamburg.de

M. Albers

E-Mail: meike.albers@hcu-hamburg.de

Dr. M. Hagemeier-Klose

E-Mail: maria.hagemeier@hcu-hamburg.de

Dr.-Ing. S. Deppisch

E-Mail: sonja.deppisch@hcu-hamburg.de
}

einer Bandbreite von alternativen Zukünften sowie eine Beurteilung der Wechselbeziehungen sowohl zwischen den relevanten Einflussfaktoren untereinander als auch zwischen den Faktoren und den Klimawandelauswirkungen. Der Szenario-Planungs-Prozess im Stadt-Umland-Raum Rostock hat gezeigt, dass die Szenariotechnik der Planung die Möglichkeit geben kann, mit zentralen Herausforderungen des Klimawandels wie Komplexität und Unsicherheit umzugehen und dies auch in die Zusammenarbeit mit lokalen und regionalen Akteuren zu integrieren. Dies wurde als eine innovative und sinnvolle Methode bewertet, die in der bisherigen Planungspraxis häufig noch nicht in dieser integrierenden Form umgesetzt wird. Die Szenarien dienen als Grundlage für die Erarbeitung konkreter Anpassungsstrategien und -maßnahmen. Der angestoßene Dialog unter den teilnehmenden Akteuren kann zudem die spätere Umsetzung von konkreten Maßnahmen erleichtern.

Schlüsselwörter Szenario-Planung · Klimawandel · Anpassung $\cdot$ Stadt- und Regionalplanung $\cdot$ Rostock

\section{Scenario Planning as a Tool for Climate Change Adaptation in Urban and Regional Planning- Key Factor Analysis and Creation of Scenarios in the Urban Region of Rostock, Germany}

\begin{abstract}
Climate change and its impacts form a new major challenge for spatial planning, as planning still shall guarantee a sustainable development in the face of complexity and uncertainty. The presented scenario planning process in the urban region of Rostock, Germany aims at adaptation to the impacts of climate change and herby realizes intensive stakeholder participation. Scenarios are elaborated on the basis of the analysis of key factors for the fu-
\end{abstract}


ture spatial development stemming from different thematic fields such as society, ecology, economics and policy and on the basis of the analysis of potential climate change impacts. These scenarios combine impacts of different severity with different potential developments of the key factors to a range of differentiated pictures of the potential future of the urban region. The scenarios allow spatial planners to consider a range of potential futures as well as interrelationships among the key factors and between the key factors and the climate change impacts. As such, they offer the possibility to cope with the main challenges of climate change, complexity and uncertainty and to integrate these challenges into a stakeholder dialogue. This was evaluated to be an innovative and sense making method, which is until now hardly implemented into current planning practice. The scenarios serve as basis for the formulation of concrete adaptation strategies and measures. Moreover, the initiated stakeholder dialogue can later facilitate the practical implementation of developed measures.

Keywords Scenario planning - Climate change · Adaptation - Urban and regional planning $\cdot$ Rostock

\section{Einführung}

Der Klimawandel und die Anpassung an seine Folgen bilden eine der zentralen Herausforderungen unserer Zeit. Die Anpassung an die Folgen des Klimawandels ist eine gesamtgesellschaftliche Aufgabe, die weder durch die Wissenschaft noch durch die Praxis allein zu bewältigen ist, gerade aufgrund der Unsicherheiten, die den Szenarien zu möglichen Auswirkungen des Klimawandels, aber auch den potenziellen Entwicklungen gesellschaftlicher Prozesse innewohnen (BACC 2008: V). Wie kann unter unsicheren Rahmenbedingungen dennoch zukunftsfähig geplant werden? Wie können hierfür Klimaänderungen mit ökologischen und gesellschaftlichen Aspekten zu einem Gesamtbild der zukünftigen Entwicklung verknüpft werden? Im Kontext nachhaltiger Stadt- und Regionalentwicklung eignet sich für die Beantwortung von Fragen wie diesen besonders ein transdisziplinärer Ansatz, der sowohl wissenschaftliche als auch praxisbezogene Perspektiven der Anpassung an die Folgen des Klimawandels integrieren kann.

Das Forschungsprojekt „plan B:altic“ beschäftigt sich mit den Auswirkungen des Klimawandels auf die Stadtund Regionalentwicklung der Küstenzone im Ostseeraum. Zusammen mit der Planungspraxis und Interessengruppen aus Stadtregionen des Ostseeraumes erarbeiten die Wissenschaftler der „HafenCity Universität Hamburg“ (HCU) stadt- und regionalplanerische Lösungsansätze zur Anpassung an die Folgen des Klimawandels.
Im Rahmen des Projekts wurde im Stadt-Umland-Raum Rostock, bestehend aus der Hansestadt Rostock und den umgebenden Gemeinden des Landkreises Rostock (vgl. Abb. 1) ein Szenario-Planungs-Prozess durchgeführt, der die Verknüpfungen von Klimaänderungen mit ökologischen und gesellschaftlichen Entwicklungen in den Betrachtungsmittelpunkt stellt und die komplexen Verflechtungen zwischen diesen berücksichtigt. Das Vorgehen anderer Ansätze, sich nur auf die Betrachtung von Klimawandelauswirkungen zu beschränken, diese mit Management-Szenarien oder mit Verfahren zu verknüpfen, die ein best case-, ein worst case- und ein business as usual-Szenario verwenden, wird in der Literatur als nicht weitreichend genug und als nicht geeignet zum Umgang mit sich wandelnden Umweltbedingungen kritisiert (vgl. O’Brian 2010: 542 ff.). Der hier verfolgte komplexe, integrative und holistische Ansatz bildet einen innovativen Gegensatz gegenüber solchen üblichen Prozessen zur Anpassung an den Klimawandel sowie den beschriebenen Szenarioansätzen im Kontext des Klimawandels.

Das Hauptziel des hier präsentierten Prozesses besteht darin, Szenarien für die Flächenentwicklung im Kontext des Klimawandels im Stadt-Umland-Raum Rostock auf der Grundlage verschiedener Bausteine zu erarbeiten und darauf aufbauend konkrete Strategien und Maßnahmen zur Anpassung an den Klimawandel zu entwickeln. Die Verwendung von Szenarien hat den Vorteil, eine Bandbreite von möglichen alternativen Zukünften und damit Unsicherheiten berücksichtigen zu können sowie die Entwicklungen der einzelnen integrierten Bausteine $\mathrm{zu}$ bündeln und mit Klimaänderungen zu verknüpfen. Für die Erarbeitung und kritische Überprüfung von Strategien und Maßnahmen können so nicht nur die derzeit „wahrscheinlichste“ oder „gewünschte“ Zukunft, sondern unterschiedliche zukünftige Entwicklungsmöglichkeiten in Betracht gezogen werden.

Ziel dieses Beitrags ist die Vorstellung der Ergebnisse des Szenario-Planungs-Prozesses. Er beschreibt die Möglichkeiten der inhaltlichen Ausgestaltung von Szenarien und deren Verwendung in der transdisziplinären Zusammenarbeit. Damit können praxisrelevante Fragen behandelt werden, beispielsweise welche Inhalte in komplexen Szenarien, die sowohl Klimawandelauswirkungen als auch gesellschaftliche und ökologische Entwicklungen verknüpfen, berücksichtigt werden können. Daher fokussiert der Beitrag die inhaltliche Auswahl und Darstellung von zentralen Bausteinen der zukünftigen Flächenentwicklung sowie die inhaltlichen Aspekte der Erstellung von Szenarien als Bilder verschiedener Zukünfte. Damit werden wertvolle Hinweise für eine praxisrelevante inhaltliche Ausgestaltung von Prozessen zur Anpassung an die Folgen des Klimawandels generiert. Auf eine theoretische Auseinandersetzung sowie eine kritische Methodenreflexion wird in diesem Beitrag verzichtet. 
Abb. 1 Stadt-Umland-Raum Rostock

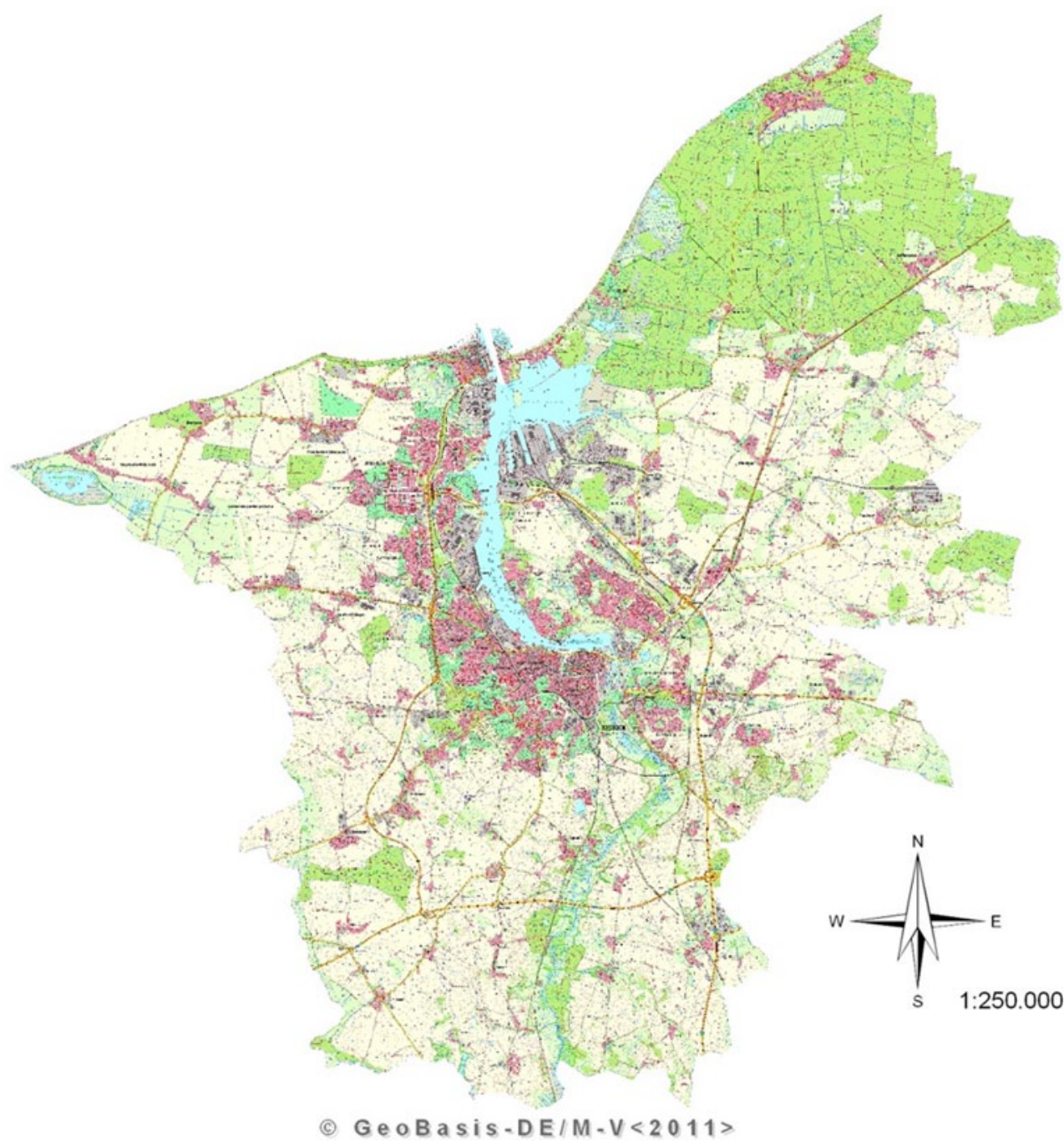

Nachfolgend werden zunächst der Klimawandel und die Anpassung an seine potenziellen Folgen im Kontext der Stadt- und Regionalplanung behandelt sowie regionalspezifische Klimaänderungen im Raum Rostock porträtiert (Kap. 2). Anschließend wird in Kap. 3 der transdisziplinäre Szenario-Planungs-Prozess näher erläutert. Kern des Beitrags bilden die Inhalte des Prozesses. Aufbauend auf Bausteinen der zukünftigen Flächenentwicklung (Kap. 4) werden die entwickelten Szenarien ausführlich in Kap. 5 dargestellt.

\section{Klimawandel und Anpassung an die Folgen des Klimawandels}

2.1 Klimawandel und Anpassung an die Folgen des Klimawandels im Kontext der Stadt- und Regionalplanung

Es besteht zunehmend Konsens in der Wissenschaft, dass sich der Klimawandel bereits heute unter anderem durch einen beschleunigten globalen Temperaturanstieg um etwa $0,19^{\circ} \mathrm{C}$ pro Dekade in den vergangenen 25 Jahren (Allison/ Bindoff/Bindschadler et al. 2009: 9) zeigt. Der Klimawandel und die Anpassung an seine Folgen stellen die Stadt- und Regionalplanung aus unterschiedlichen Gründen vor große Herausforderungen. Die konkreten künftigen Auswirkungen des Klimawandels und die Klimaszenarien sind aufgrund mehrfacher Unsicherheitsquellen und Zukunftskonstruktionen mit großen Unsicherheiten verbunden. Insbesondere für regionale Betrachtungen der Klimaänderungen besteht die Schwierigkeit, die globalen Szenarien auf eine kleinräumigere Ebene zu übertragen, da diese Klimaentwicklungen teilweise sogar dem globalen Trend widersprechen können (Daschkeit/Sterr 2005: 268). Zudem werden zukünftige globale Klimaentwicklungen häufig mittels Szenarien des Intergovernmental Panel on Climate Change (IPCC, Zwischenstaatlicher Ausschuss für Klimaänderungen) beschrieben, die meist bis zum Jahr 2100 reichen (vgl. IPCC 2007). Für Praktiker sind jedoch kürzere Zeiträume von größerem Interesse, da Planungsinstrumente häufig kurzfristiger kon- 
zipiert sind und Ziele der Stadt- und Regionalplanung häufig nur für die nähere Zukunft formuliert werden. Überdies können sich lokale und regionale Änderungen der Landnutzung, Infrastruktur sowie in der Wirtschaftsentwicklung stark auf regionalklimatische Verhältnisse auswirken, sich somit schlimmstenfalls mit dem globalen Klimawandel überlagern und ihn damit verstärken. Beispielsweise könnten Frischluftschneisen, die zur Kühlung von Innenstädten beitragen sollten, der Nachverdichtung zum Opfer fallen, womit die innerstädtische Erwärmung zusätzlich gefördert würde. Andererseits kann den Folgen auf regionaler Ebene gezielt entgegengesteuert werden. Um das vorhergehende Beispiel erneut aufzugreifen, wäre es bei schrumpfenden Städten möglich, neue Frischluftschneisen gezielt durch Abriss von Gebäuden zu schaffen.

Hieraus ergibt sich die Notwendigkeit, sich auch im Kontext der Stadt- und Regionalplanung an potenziell negative Folgen des Klimawandels anzupassen sowie gegebenenfalls entstehende Chancen zu nutzen, um eine nachhaltige und zukunftsfähige Entwicklung gewährleisten zu können. Durch eine rechtzeitige Anpassung ist die Gesellschaft in der Lage, insbesondere mit den in Zukunft verschärften Gefahren des Klimawandels umgehen zu können, also zu handeln, bevor Schäden eintreten (Stehr/von Storch 2008: 270). Die Forschung zeigt, dass es bei sogenannten schleichenden Katastrophen, wozu auch der Klimawandel gerechnet wird, entscheidend ist, wann eine Bedrohung erkannt wird und wie schnell entsprechende Maßnahmen zur Anpassung umgesetzt werden (Buttschardt 2010: 19).

Die Raumplanung spielt eine besondere Rolle bei der Anpassung an die potenziellen Folgen des Klimawandels, obwohl in der bisherigen Praxis die Bedeutung und Umsetzung von Maßnahmen der Anpassung deutlich hinter denen des Klimaschutzes zurückblieben (Davoudi 2009: 8 f.). Beispielsweise können Entscheidungen über zukünftige Landnutzungsänderungen oder Bebauungen vor dem Hintergrund des Meeresspiegelanstiegs oder vermehrter sowie verstärkter Flusshochwässer hinterfragt werden (Ritter 2007: 533). Die Raumplanung spielt zudem eine herausragende Rolle, weil sie sowohl die Gestalt und Lage von Siedlungsflächen, Infrastruktur und Grünflächen beeinflusst (BMVBS/BBSR 2009: 24 f.) als auch Ansprüche an die Landnutzung in einem bestimmten Gebiet von verschiedenen Akteuren und Sektoren integrieren muss.

Wie oben skizziert, stellen sich mit dem Klimawandel besondere Herausforderungen für die Planung, da sich durch Unsicherheiten der Klimaszenarien und weiterer künftiger Entwicklungen und deren Wechselbeziehungen und den langen Zeithorizont Entscheidungs- und Abwägungsspielräume auftun, die noch nicht ausgefüllt sind (Böschen/Weis 2007: 183). Errechnete Klimaszenarien zeigen meist mögliche Änderungen bis zum Ende des 21. Jahrhunderts, während sich Planungszeiträume oft nur auf die nächsten zehn bis 15
Jahre fokussieren (Fleischhauer/Bornefeld 2006: 169; Ritter 2007: 537). In der Raumplanung hat die Auseinandersetzung mit zukünftigen Entwicklungen und deren Unsicherheiten zwar bereits eine lange Tradition, der Klimawandel scheint diesen Aspekt jedoch noch deutlich zu verstärken (Heiland/ Kowarik 2008: 421). Da planerische Entscheidungen Städte und Regionen über sehr lange Zeiträume beeinflussen, müssen Gebäude und Infrastrukturen bereits heute sowohl an derzeitige als auch an zukünftige potenzielle Klimawandelfolgen angepasst werden (Hallegatte 2009).

\subsection{Klimawandel in der Region Rostock}

Der Stadt-Umland-Raum Rostock liegt an der Ostseeküste Mecklenburg-Vorpommerns. Die Hansestadt Rostock als Kernstadt hat etwa 200.000 Einwohner. Der Hafen und die nachgelagerte maritime Wirtschaft sowie der Tourismus sind die zentralen Wirtschaftszweige. Gerade in Küstennähe befindet sich in der gesamten Region viel touristische Infrastruktur und dadurch bedingt hoher Nutzungsdruck. Neben stark verdichteten städtischen Siedlungs- und Gewerbegebieten finden sich Plattenbausiedlungen, Einfamilien- und Ferienhaussiedlungen sowie Kleingartenanlagen. Auch im Stadtgebiet sind weitläufige Grünflächen vorhanden. Zudem bestehen im Stadtgebiet und im Umland größere Naturschutzgebiete. Insbesondere die küstennahen Niederungen und Moore sind hier herauszustellen, ebenso wie die Rostocker Heide oder die Auen des Flusses Warnow, aus dem auch die Versorgung mit Trinkwasser für die Hansestadt Rostock erfolgt (vgl. Amt für Raumordnung und Landesplanung Mittleres Mecklenburg/Rostock 2011). Vor dem Hintergrund der potenziellen Folgen des Klimawandels sind die Ausstattung mit städtischen Grünflächen und Frischluftschneisen sowie großflächige Wald- und Naherholungsgebiete von besonderer Bedeutung, da sie zur Kühlung bei Hitze beitragen, im Gegensatz zu versiegelten Flächen Wasser bei Starkregenereignissen aufnehmen können und der Bevölkerung als Erholungsraum dienen (Fleischhauer/ Bornefeld 2006: 166; Greiving/Fleischhauer 2008: 64).

Im Zuge des Klimawandels wird es in der Region Rostock möglicherweise zu einer Erhöhung der durchschnittlichen Jahresmitteltemperaturen im Vergleich zur derzeitigen Referenzperiode 1961-1990 kommen, wobei die Änderungen voraussichtlich im Winterhalbjahr am deutlichsten sein werden. Im vergangenen Jahrhundert hat sich die Jahresdurchschnittstemperatur im südlichen Ostseeraum bereits um etwa $0,07^{\circ} \mathrm{C}$ pro Dekade erhöht (BACC 2008: 23). Gemäß verschiedener Szenarien sind bis zum Jahr 2050 Erhöhungen von $1^{\circ} \mathrm{C}$ bis über $2^{\circ} \mathrm{C}$ möglich (HelmholtzZentrum Geesthacht 2011: o. S.).

Weiterhin werden Änderungen der jährlichen Niederschlagsmenge und der jahreszeitlichen Verteilung erwartet. Im 20. Jahrhundert zeigten sich unterschiedliche Trends 
zur Niederschlagsmenge in Mecklenburg-Vorpommern, die Entwicklungen deuten aber auf trockenere Sommer und feuchtere Winter hin. Zukünftig (bis zum Jahr 2050) kann man von einer Erhöhung der Jahresniederschlagsmengen um 2 bis $10 \%$, trockeneren Sommern und feuchteren Wintern ausgehen (Helmholtz-Zentrum Geesthacht 2011: o. S.).

Durch Landabsenkungen in Verbindung mit globalen Temperaturerhöhungen kommt es zur Anhebung des mittleren Meeresspiegelniveaus. Dieser Anstieg betrug an der Station Warnemünde nach Messungen von 1855 bis 2009 etwa 1,2 mm pro Jahr (NOAA 2012: o. S.) und war vorwiegend auf die Absenkung der Landmasse zurückzuführen. In den vergangenen Jahrzehnten konnte eine Beschleunigung festgestellt werden (BACC 2008: 97) und so könnte es zusammen mit einer temperaturbedingten Meeresspiegelerhöhung nach aktuellem Wissensstand bis zum Ende des Jahrhunderts an den südlichen Ostseeküsten zu einem Anstieg des Meeresspiegels von bis zu einem Meter kommen (Meier/Bromann/Kjellström 2004: 66).

Eine besonders hohe Bedeutung kommt der Entwicklung der Häufigkeit und Intensität von Extremwetterereignissen zu, da diese starke negative Konsequenzen haben können. Während in der Vergangenheit eine Zunahme der „warmen Extreme“ wie Hitzetage zu beobachten war, nahm die Anzahl „kalter Extreme“ wie etwa die der Eistage ab (BACC 2008: 61). Eine Zunahme der Häufigkeit und Intensität von Starkniederschlägen in Wintern konnte beobachtet werden (BACC 2008: 65). Kein signifikanter Trend konnte bisher bei Änderungen des Auftretens von Stürmen erkannt werden (Bärring/von Storch 2004: 1). In Bezug auf die Zunahme „warmer" und die Abnahme „kalter Extreme“ sowie die Zunahme von Starkniederschlägen wird davon ausgegangen, dass sich diese Trends weitestgehend fortsetzen und verstärken werden. Je nach Klimaszenario ist auch eine leichte Zunahme von Stürmen und deren Intensitäten möglich (BACC 2008: 178).

Neben den skizzierten potenziellen Auswirkungen des Klimawandels im Raum Rostock spielt die Betroffenheit und Wahrnehmung des Klimawandels durch unterschiedliche gesellschaftliche Akteure eine zentrale Rolle für die Formulierung und Umsetzung von Anpassungsstrategien und Anpassungsmaßnahmen. So werden der Klimawandel und seine Folgen mit potenziellen Schadensereignissen von verschiedenen Bevölkerungsgruppen, politischen Entscheidungsträgern, Wissenschaftlern, Planungs- und Verwaltungsinstitutionen und weiteren Akteuren unterschiedlich wahrgenommen, eingeschätzt und als Problem definiert (vgl. auch Overbeck/Hartz/Fleischhauer 2008: 367). Der im Folgenden beschriebene transdisziplinäre Szenario-Planungs-Prozess eignet sich besonders, um unterschiedliche Sichtweisen sowohl in die Entwicklung von Szenarien als auch in die Erarbeitung von Anpassungsstrategien und -maßnahmen zu integrieren.

\section{Der Prozess der Anpassung an die Folgen des Klimawandels im Stadt-Umland-Raum Rostock}

\subsection{Praxis-Wissenschafts-Dialog}

Der Prozess der Anpassung an die Folgen des Klimawandels im Stadt-Umland-Raum Rostock stand unter dem Motto „Zukunft aktiv gestalten“. Dieser Prozess wurde von der Forschungsgruppe ,plan B:altic“ initiiert, um einen Dialog zur zukünftigen Flächenentwicklung vor dem Hintergrund des Klimawandels mit der Planungspraxis anzustoßen. Mit dem Prozess wurde das Ziel verfolgt, die Auswirkungen des Klimawandels auf die Flächenentwicklung im Stadt-Umland-Raum zu betrachten und gemeinsam mögliche Strategien zur Anpassung an die Folgen des Klimawandels zu entwickeln. Dabei ging es darum, Handlungsmöglichkeiten für die Planung zur Gestaltung der räumlichen Entwicklung aufzuzeigen und zu diskutieren. Dies ist jedoch nicht nur Aufgabe der Politik oder der Verwaltung, sondern erfordert die Mitwirkung weiterer regionaler und lokaler Akteure, Institutionen und der Öffentlichkeit.

Der Prozess, der eine dreistufige Workshop-Reihe umfasste, wurde in intensiver Kooperation mit einer Kerngruppe konzipiert und durchgeführt, die aus verschiedenen Verwaltungsinstitutionen aus dem Raum Rostock zusammengesetzt ist. Der Kerngruppe gehörten Vertreter der Hansestadt Rostock, des Regionalen Planungsverbands Mittleres Mecklenburg/Rostock, des Landkreises Rostock, des Staatlichen Amts für Landwirtschaft und Umwelt Mittleres Mecklenburg und der HafenCity Universität Hamburg (HCU) an.

$\mathrm{Zu}$ den drei Szenario-Workshops, die jeweils einen Tag dauerten, wurden weitere, über die Kerngruppe hinausgehende Teilnehmer aus Wissenschaft, Wirtschaft, verschiedenen Verwaltungsinstitutionen, Naturschutzverbänden sowie der Politik eingeladen. Es wurde darauf geachtet, eine möglichst gute Mischung von Akteuren zu erreichen und alle relevanten Bereiche abzudecken, um die Integration möglichst vieler Perspektiven zu gewährleisten. Hierzu wurde gemeinsam mit der Kerngruppe eine Stakeholder-Analyse durchgeführt. Von etwa 100 persönlich eingeladenen Akteuren konnten jeweils zwischen 30 und 40 Personen für die Szenario-Workshops gewonnen werden.

\subsection{Ablauf des Prozesses}

In diesem Abschnitt wird der Ablauf des gesamten Szenario-Planungs-Prozesses zusammengefasst vorgestellt, ausführlichere Darstellungen zu den inhaltlichen Arbeitsschritten erfolgen in den anschließenden Kapiteln. Der Szenario-Planungs-Prozess verlief in fünf aufeinander aufbauenden Phasen (vgl. Abb. 2; vgl. zur Szenariotechnik: von Reibnitz 1992; Stiens 1998; Fink/Siebe 2006; Scholles 2008; Mietzner 2009). Aufbauend auf der Szenariofeldbe- 
Abb. 2 Ablaufschema des Szenario-Planungs-Prozesses. (Grafik: Maria Hagemeier-Klose, Meike Albers)

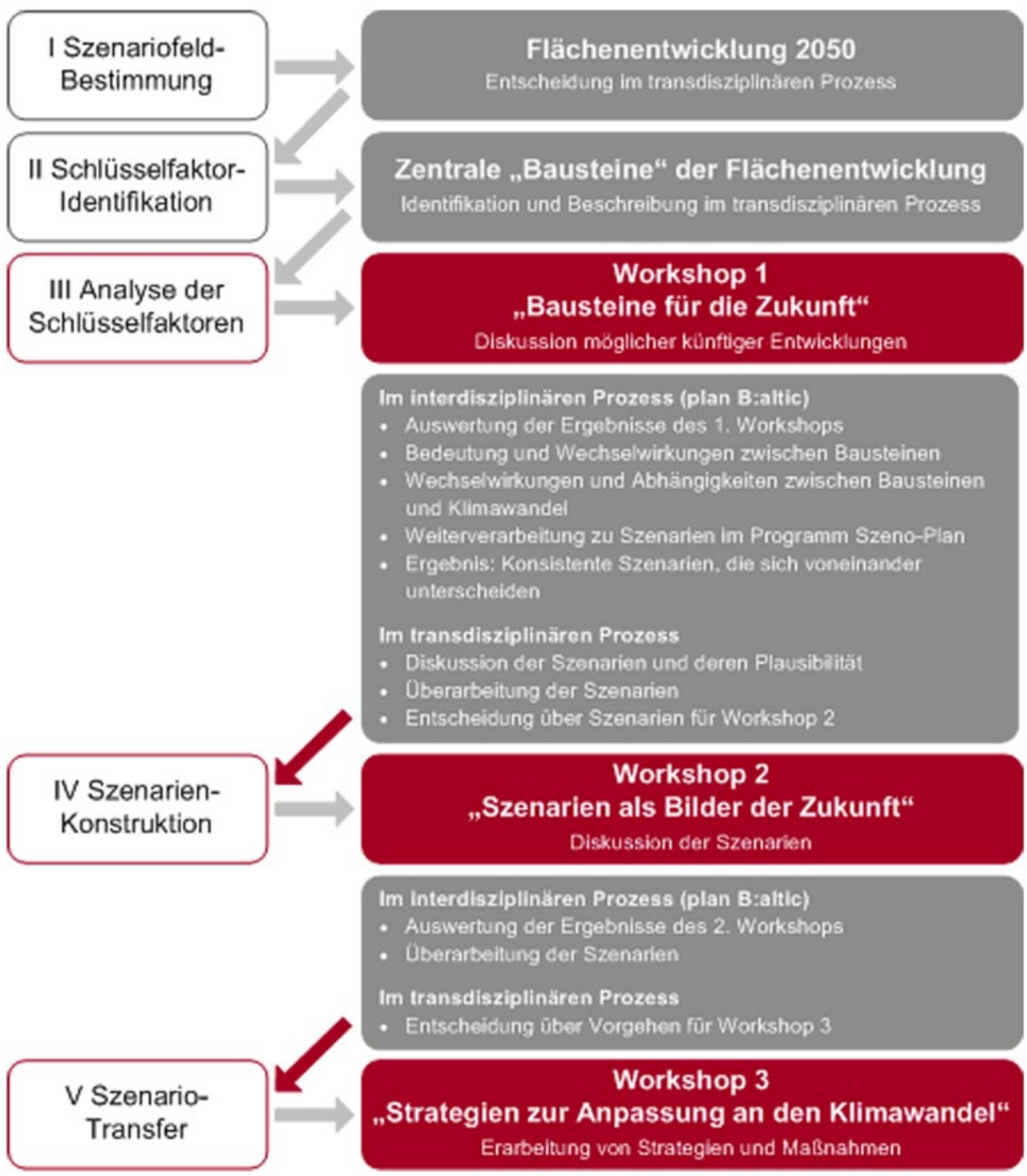

stimmung (vgl. Abb. 2, Phase I) sowie der Identifikation und Analyse von Bausteinen (vgl. Abb. 2, Phasen II Schlüsselfaktor-Identifikation und III Analyse der Schlüsselfaktoren) wurden Szenarien für die zukünftige Entwicklung konstruiert (vgl. Abb. 2, Phase IV Szenarienkonstruktion), die unterschiedliche mögliche Zukünfte aufzeigen. Der anschließende Transfer dieser Szenarien (vgl. Abb. 2, Phase V Szenariotransfer) diente der Entwicklung konkreter Strategien und Maßnahmen zum Umgang mit den dargestellten Entwicklungen. Kernelement des Szenario-Planungs-Prozesses waren drei Workshops, die jeweils an die besonders relevanten Phasen angeschlossen waren.

Die erste Phase der Szenariofeldbestimmung, in der es um die Abgrenzung der inhaltlichen Ausrichtung der Szenarien geht, stellt den Beginn jedes Szenario-Planungs-Prozesses dar. Als Rahmen wurde die Anpassung an die Folgen des Klimawandels im Kontext der Stadt- und Regionalplanung vorab festgesetzt. Im transdisziplinären Dialog mit der
Kerngruppe wurde das Szenariofeld auf die Flächenentwicklung im Stadt-Umland-Raum Rostock bis zum Jahr 2050 fokussiert. Durch diese Fokussierung war es möglich, das Feld der relevanten Bausteine einzugrenzen und zielgerichtet im Praxis-Wissenschafts-Dialog zu diskutieren. Auch die Phase II, die Identifikation der Bausteine, sowie die Phase III, die erste Analyse der Bausteine, erfolgten im transdisziplinären Austausch gemeinsam mit der Kerngruppe (vgl. Kap. 3). Die vier Bausteine aus dem Bereich Klimaänderungen wurden durch die Forschungsgruppe vordefiniert. Es wurden der Meeresspiegelanstieg, die Erhöhung der Jahresmitteltemperatur, Änderungen im Niederschlagsgeschehen sowie eine Zunahme von Extremwetterereignissen festgelegt. Als soziale/gesellschaftliche Bausteine wurden Bevölkerungsstruktur und Wohnen identifiziert. Wirtschaftliche Bausteine bildeten der Hafen, Tourismus und Handel sowie Gewerbe. Im Bereich der Politik erfolgte eine Einigung auf die Bausteine formelle Instrumente, Verhältnis zwischen 
Stadt und Umland, politische Prioritäten, Finanzsituation und Energiewende. Als ökologische Bausteine wurden die Freiraumstruktur und -qualität, Umweltgüter sowie Landund Forstwirtschaft festgelegt.

Die Analyse und Diskussion dieser Bausteine war Gegenstand des ersten Szenario-Workshops „Bausteine für die Zukunft“" im April 2011. Gemeinsam mit der Kerngruppe wurde zu jedem Faktor ein Trendblatt erarbeitet, das sowohl die Bedeutung des Faktors als auch die bisherige Entwicklung sowie mögliche zukünftige Entwicklungspfade bis zum Jahr 2050 beinhaltete. Im Rahmen des Workshops wurden die Klimawandel-Bausteine gemeinsam mit Bausteinen der ökologischen und gesellschaftlichen Entwicklung mit 40 Teilnehmenden diskutiert. Neben Entwicklungspfaden der einzelnen Bausteine wurden explizit Wechselwirkungen zwischen den Bausteinen sowie die Einflüsse des Klimawandels thematisiert.

Die Analyse der Bausteine diente als Basis für Phase IV, die Konstruktion der Szenarien (vgl. Kap. 4). In die Konstruktion flossen Bewertungen der Bedeutung, die Wechselwirkungen zwischen den Bausteinen sowie die jeweils unterschiedlichen Entwicklungspfade ein. Dadurch ermöglichten die Szenarien sowohl die Darstellung der komplexen Zusammenhänge als auch die Integration von Dynamik. Die Konstruktion und eine Vorauswahl von Szenarien erfolgten durch die Forschungsgruppe ,plan B:altic“ (vgl. Kap. 4 zur genaueren Beschreibung der Szenarienkonstruktion). Für die Entscheidung zur endgültigen Auswahl der Szenarien wurden sechs Rohszenarien ausgewählt und anschließend mit der Kerngruppe diskutiert. Die Auswahl erfolgte anhand der Kriterien Konsistenz, Unterscheidbarkeit, Interpretierbarkeit und Darstellung einer Bandbreite von möglichen Zukünften. Anhand der Diskussion mit der Kerngruppe wurde die Auswahl auf vier Szenarien reduziert, die unterschiedliche Zukünfte für die Flächenentwicklung im Stadt-Umland-Raum Rostock präsentieren. Diese Szenarien wurden hinsichtlich Plausibilität, Empfindlichkeit gegenüber unerwarteten Ereignissen sowie ihrer Flächenwirkung im Rahmen des zweiten Szenario-Workshops „Szenarien als Bilder der Zukunft“ im November 2011 mit 30 Teilnehmenden diskutiert. Anhand der Diskussionsergebnisse wurden die Szenarien für die fünfte und abschließende Phase, den Transfer in Strategien zur Anpassung an den Klimawandel, aufbereitet, die das Thema des dritten Szenario-Workshops im April 2012 waren.

\section{Bausteine der Flächenentwicklung bis 2050}

\subsection{Die Bestimmung des Szenariofeldes für den Szenario- Planungs-Prozess}

Das Thema der Anpassung an die Folgen des Klimawandels hat sehr viele unterschiedliche Facetten, die im Rahmen eines
Szenario-Planungs-Prozesses betrachtet werden können. So könnten beispielsweise die wirtschaftliche Entwicklung, die Biodiversität oder der Katastrophenschutz im Vordergrund stehen. Um zielgerichtet diskutieren zu können, ist es deshalb erforderlich, die Ausrichtung des Szenario-PlanungsProzesses klar zu definieren und sowohl einen inhaltlichen als auch einen räumlichen Schwerpunkt festzulegen.

Der Fokus des hier vorgestellten Szenario-PlanungsProzesses wurde auf die Stadt- und Regionalplanung gelegt, die die räumlich integrierende Gesamtplanung bilden, die im Wesentlichen die unterschiedlichen Ansprüche an die Ressource Fläche sowie deren Funktionen, Nutzungsarten und -intensitäten sowie weitere Entwicklung (und Vorsorge) zu steuern beabsichtigt. Aus diesem Grund erfolgte die Fokussierung auf die Entwicklung der Fläche, also auf alle räumlichen Nutzungen. Darunter fallen sowohl bebaute als auch nicht bebaute Flächen. Dabei werden nicht nur bestimmte inhaltliche Themen (z. B. Tourismus oder Wirtschaft) betrachtet, sondern alle räumlichen Nutzungen, die durch die Stadt- und Regionalplanung beeinflusst werden können. In Abstimmung mit der Kerngruppe wurde als Betrachtungsraum der Stadt-Umland-Raum Rostock ausgewählt.

Das Ziel des Szenario-Planungs-Prozesses bestand folglich darin, im Kontext des Klimawandels Szenarien für die Flächenentwicklung im Stadt-Umland-Raum Rostock zu erarbeiten und darauf aufbauend Strategien und Maßnahmen zum Umgang mit den potenziellen Folgen des Klimawandels zu entwickeln.

\subsection{Die Identifikation und Analyse der Bausteine}

Die Entwicklung der Flächen wird von vielen unterschiedlichen Bausteinen beeinflusst, die für den Szenario-Planungs-Prozess identifiziert werden müssen. Da es sich bei der Flächenentwicklung um einen sehr komplexen Prozess handelt, konnten im Rahmen des Szenario-Planungs-Prozesses (auch aufgrund der zeitlich und personell begrenzten Ressourcen) nicht alle Bausteine, die Einfluss auf die Flächenentwicklung haben, berücksichtigt werden. Deshalb mussten Bausteine ausgewählt werden, die als besonders wichtig für die Flächenentwicklung angesehen werden. Dazu wurde zunächst von wissenschaftlicher Seite eine Liste möglicher Bausteine vorbereitet, für die auf Literatur und allgemeine Informationen über die Region Rostock zurückgegriffen wurde. Im nächsten Schritt wurde dieser Vorschlag mit der Kerngruppe diskutiert. Dabei stand die Frage im Vordergrund, welche Bausteine nach Einschätzung der Experten vor Ort die größte Bedeutung für die Flächenentwicklung haben.

Da das Ziel darin bestand, mit Hilfe der Szenarien unterschiedliche Entwicklungen und ,alternative Zukünfte“ abzubilden, wurden auch für die einzelnen Bausteine unter- 
schiedliche Entwicklungen zugrunde gelegt. Es wurde also nicht betrachtet, welche Entwicklung der einzelnen Bausteine zum derzeitigen Zeitpunkt am wahrscheinlichsten angesehen wird, sondern welche unterschiedlichen Entwicklungen grundsätzlich denkbar sind. Für jeden Baustein wurden zwei bis vier verschiedene Entwicklungspfade beschrieben, die eine möglichst große Bandbreite für die zukünftige Entwicklung dieses Faktors widerspiegeln. Damit werden Dynamiken in der Entwicklung der Bausteine ebenso berücksichtigt wie Entwicklungsmöglichkeiten, die zwar nach dem derzeitigen Trend weniger wahrscheinlich, aber dennoch denkbar sind. Für die Ableitung der Entwicklungspfade wurde auf vorhandene Studien über künftige Entwicklungen, Erfahrungen und Einschätzungen der lokalen Akteure aus vergangenen Entwicklungen sowie (für die klimatischen Veränderungen) auf Klimaszenarien zurückgegriffen. Darauf aufbauend wurden die Entwicklungspfade qualitativ beschrieben und sowohl mit der Kerngruppe als auch im ersten Szenario-Workshop mit den Teilnehmenden diskutiert. Dabei standen zwei Fragen im Vordergrund:

- Welche Bausteine sind aus Sicht der Teilnehmenden für die Flächenentwicklung von Bedeutung?

- Wie können sich die einzelnen Bausteine bis zum Jahr 2050 entwickeln?

Auf der Grundlage der Ergebnisse des ersten Szenario-Workshops wurden insgesamt 18 Bausteine mit unterschiedlichen zukünftigen Entwicklungsmöglichkeiten identifiziert, die sich in fünf Handlungsfelder gliedern lassen:

- Klimaänderungen,

- soziale/gesellschaftliche Aspekte,

- ökologische Aspekte,

- ökonomische Aspekte sowie

- politische Aspekte.

\subsection{Die Bausteine und ihre Entwicklungsmöglichkeiten}

Im Folgenden werden zunächst die einzelnen Bausteine, die im Rahmen des Szenario-Planungs-Prozesses berücksichtigt wurden, mit ihren jeweiligen zukünftigen Entwicklungsmöglichkeiten bis zum Jahr 2050 aufgeführt und beschrieben.

\subsubsection{Klimaänderungen}

Kontinuierlicher Anstieg des Meeresspiegels. Das mittlere Niveau des Meeresspiegels hat große Bedeutung für die Flächenentwicklung in tiefliegenden Bereichen der Küstenregionen und kann bestehende und zukünftig geplante Nutzungen beeinflussen.

Entwicklungsmöglichkeiten: Meeresspiegelanstieg um 1) 20,2$) 60$ oder 3) $100 \mathrm{~cm}$.
Anstieg der durchschnittlichen Jahresmitteltemperatur. Der Klimawandel geht voraussichtlich mit einer Veränderung der durchschnittlichen Jahresmitteltemperatur einher. Diese kann direkte und indirekte Einflüsse auf die Flächenentwicklung haben.

Entwicklungsmöglichkeiten: Anstieg der Jahresmitteltemperatur um etwa 1$) 1{ }^{\circ} \mathrm{C}$, 2) $1,6^{\circ} \mathrm{C}$ oder 3$) 2,2{ }^{\circ} \mathrm{C}$.

Veränderungen der jährlichen Niederschlagsmenge und saisonalen Verteilung. Es wird davon ausgegangen, dass sich die jährliche Niederschlagsmenge und die saisonale Verteilung künftig verändern werden. Diese Veränderungen können sich je nach Flächenentwicklung unterschiedlich auswirken.

Entwicklungsmöglichkeiten: Anstieg der Niederschlagsmenge um etwa 1) $1 \%$, 2) $6 \%$ oder 3) $10 \%$.

Zunahme der Häufigkeit und Intensität von Extremwetterereignissen. Als Extremwetterereignisse können sowohl Einzelereignisse wie Sturm oder Starkniederschlag als auch Perioden wie Hitzewellen und Dürren bezeichnet werden. Diese können definiert werden durch relativ seltenes Auftreten, hohe Intensität oder Dauer und relativ hohe Schäden.

Entwicklungsmöglichkeiten: 1) geringer, 2) mittlerer oder 3) starker Anstieg von Extremwetterereignissen.

\subsubsection{Soziale/Gesellschaftliche Aspekte}

Wohnen und Gemeinbedarf. Die Nachfrage nach Wohnraum und der Anteil an Gemeinbedarfsgebäuden in Wohngebieten sowie ihre Entwicklung haben direkten Einfluss auf die Flächeninanspruchnahme.

Entwicklungsmöglichkeiten: 1) Wohnungsrückbau, 2) Wohnungsumbau, 3) Wohnungsneubau im Umland/Suburbanisierung, 4) Wohnungsneubau in Rostock/Reurbanisierung.

Bevölkerungsstruktur und Bevölkerungsentwicklung. Die Bevölkerungsstruktur und ihre Entwicklung beeinflussen die Flächenentwicklung direkt über den Bedarf an Wohnbauflächen, Infrastruktureinrichtungen, Flächen mit Erholungsfunktion und Nachfrage nach Handel und Dienstleistungen.

Entwicklungsmöglichkeiten: 1) Bevölkerungsrückgang in Rostock/Suburbanisierung, 2) Bevölkerungsrückgang im Gesamtraum, 3) Bevölkerungswachstum in Rostock/ Reurbanisierung, 4) Bevölkerungswachstum in Rostock und Umland.

Verkehrsinfrastruktur und Verkehrsentwicklung. Verkehrsinfrastruktur und Verkehrsentwicklung prägen die Flächennutzung direkt durch Flächeninanspruchnahme. Dabei sind sowohl der motorisierte Individualverkehr, der öffentliche Personennahverkehr und der Güterverkehr von Bedeutung. 
Entwicklungsmöglichkeiten: 1) Ausbau der Verkehrsfläche für den motorisierten Individualverkehr, 2) regionales Mobilitätskonzept/Verlagerung auf den öffentlichen Personennahverkehr, 3) Ausbau der Verkehrsfläche für Güterverkehr.

\subsection{3 Ökologische Aspekte}

Umweltgut Wasser. Die Qualität und Verfügbarkeit von Wasser (Grundwasser, Trinkwasser, Badewasser) ist abhängig vom Flächenverbrauch und der Intensität der Flächennutzung. Das Umweltgut Wasser hat direkten Einfluss auf die Flächenentwicklung, da es die potenzielle Nutzbarkeit von Flächen bestimmt.

Entwicklungsmöglichkeiten: 1) Stagnation oder Rückgang der Wasserqualität, 2) Rückgang der Wasserqualität durch Oberflächenabfluss, 3) Trockenperioden.

Freiraumstruktur, Freiraumentwicklung und Qualität der Freiräume. Sowohl die Struktur und Entwicklung der Freiräume als auch deren Qualität und Klassifizierung (z. B. FFH-Gebiete oder Naturschutzgebiete) prägen die Flächenentwicklung im Raum Rostock entscheidend mit.

Entwicklungsmöglichkeiten: 1) Rückgang von Freiflächen, 2) Umsetzung der Landschaftsschutzziele, 3) Erhalt bestehender Freiräume, 4) Landschaftszerschneidung und Qualitätsverlust.

\subsection{4 Ökonomische Aspekte}

Tourismus. Die Region Rostock gehört zu den beliebtesten Urlaubsregionen Deutschlands und der Tourismus ist ein wichtiger Wirtschaftsfaktor, weshalb eine Vielzahl von überregional bedeutsamen touristischen Zentren existiert.

Entwicklungsmöglichkeiten: 1) Anstieg des Sommertourismus, 2) Ausbau des Wintertourismus, 3) umweltverträgliche Urlaubsformen.

Hafen und maritime Wirtschaft. Der Hafen und die maritime Wirtschaft sind eine strukturbestimmende Branche. Die Flächenverfügbarkeit ist hier bereits begrenzt, so dass zur Erfüllung der Industrie- und Logistikfunktion weitere Flächen benötigt werden.

Entwicklungsmöglichkeiten: 1) positive Entwicklung und Ausbau des Hafens, 2) Stagnation ohne neue Flächeninanspruchnahme, 3) Bedeutungsrückgang des Hafens.

Handels- und Gewerbeentwicklung. Handels- und Gewerbestandorte stellen wesentliche Elemente der Flächenentwicklung dar. Sie nehmen direkt Flächen in Anspruch, haben aber auch Auswirkungen auf Verkehrsströme, den Arbeitsmarkt und Steuereinnahmen der Kommunen.
Entwicklungsmöglichkeiten: 1) Konzentration auf Rostock und verfügbare Flächenreserven, 2) Stagnation, 3) starkes Wachstum in Rostock und Umland.

Land- und Forstwirtschaft. Im Raum Rostock existieren zahlreiche land- und forstwirtschaftliche Flächen, die sowohl wirtschaftlichen, aber auch ökologischen und Erholungsfunktionen dienen.

Entwicklungsmöglichkeiten: 1) Stagnation, Extensivierung und Biolandbau, 2) gegenläufige Entwicklungen, teils Extensivierung und teils Intensivierung, 3) Stagnation und Intensivierung.

\subsubsection{Politische Aspekte}

Finanzsituation. Die Finanzsituation Rostocks und der Umlandgemeinden hat Einfluss auf die jeweilige kommunale Handlungsfähigkeit und damit indirekt Einfluss auf die Flächenentwicklung oder die Bereitstellung von Infrastruktur.

Entwicklungsmöglichkeiten: 1) Verschlechterung der Finanzsituation, 2) Verbesserung der Finanzsituation, 3) Priorität für Schuldenabbau.

Politische Prioritäten der Stadt-, Regional- und Umlandentwicklung. Die tatsächliche Entwicklung ist stark abhängig von den politischen Zielsetzungen und Prioritäten, insbesondere auf kommunaler und auf Landesebene. Einfluss auf die Flächenentwicklung nehmen darüber hinaus auch Interessen- und Lobbygruppen sowie die lokale Bevölkerung.

Entwicklungsmöglichkeiten: 1) nachhaltige Stadt- und Regionalentwicklung, 2) Dominanz wirtschaftlicher Interessen, 3) häufig wechselnde politische Mehrheiten, 4) Dominanz ökologischer Ziele.

Verhältnis zwischen Stadt und Umland. Die Entwicklung des Raumes Rostock und die Umsetzung des gemeinsamen Entwicklungsrahmens hängen sehr vom derzeitigen und künftigen Verhältnis zwischen der Hansestadt Rostock und den Umlandgemeinden ab.

Entwicklungsmöglichkeiten: 1) Stärkung der Stadt-Umland-Kooperation, 2) konkurrierendes Verhältnis, 3) Fusion von Rostock und Umlandgemeinden.

Formelle Instrumente und Prozesse. Die verfügbaren formellen Instrumente und Prozesse der Stadt- und Regionalplanung treffen Aussagen zur räumlichen Entwicklung und geben so den Rahmen für die Entwicklung vor.

Entwicklungsmöglichkeiten: 1) ausgewogene Berücksichtigung aller raumrelevanten Aspekte, 2) wirtschaftliche Themen im Vordergrund. 
Energetische Selbstversorgung/Energiewende. Die Ziele der kommunalen Selbstversorgung im Bereich Energie und eine Umstellung auf erneuerbare Energien zum Klimaschutz (Energiewende) können erhebliche Auswirkungen auf Flächennutzungen haben (u. a. Anlagenbau, Flächennutzung für erneuerbare Energien).

Entwicklungsmöglichkeiten: 1) starke Zunahme regenerativer Energien, 2) konstanter Anteil regenerativer Energien, 3) mäßige Zunahme regenerativer Energien, 4) $100 \%$ regenerative Energien.

Die Bausteine mit ihren jeweiligen Entwicklungsmöglichkeiten bildeten die Grundlage für die im Folgenden dargestellte Erstellung der Szenarien.

\section{Bilder der Zukunft - vier Szenarien zur zukünftigen Flächenentwicklung im Stadt-Umland-Raum Rostock}

Die ausgewählten Szenarien zeichnen vier unterschiedliche Bilder der Zukunft. Szenarien sind keine Prognosen, also keine Vorhersagen der Zukunft, sondern stellen mögliche, alternative Zukünfte dar (Peterson/Cumming/Carpenter 2003: 360). Sie präsentieren damit ein hypothetisches Bild der Zukunft und weisen auf mögliche Entscheidungs- oder Ansatzpunkte für Strategien hin (Kahn/Wiener 1967: 6; von Reibnitz 1992: 14). Unter diesen Prämissen können Szenarien eine Bandbreite möglicher Zukünfte aufzeigen und mögliche zukünftige Entwicklungen bündeln. Szenarien dienen der Identifikation von Entscheidungspunkten und Handlungsoptionen, um daraus adäquate Strategien abzuleiten, da sie auch eine Analyse der Konsequenzen bestimmter Entscheidungen und Handlungen ermöglichen (Peterson/Cumming/Carpenter 2003: $360 \mathrm{ff}$.). Ein weiterer Vorteil bei der Verwendung von Szenarien ist, dass sie nicht nur eine, also die „wahrscheinlichste“ oder ,gewünschte“ Zukunft zeigen, sondern sehr unterschiedliche mögliche Entwicklungen. Dadurch können Strategien und Maßnahmen vor dem Hintergrund der Unsicherheit über unterschiedliche künftige Entwicklungen überprüft und diskutiert werden. So können etwa der Einfluss der Klimaän- derungen oder die Empfindlichkeit gegenüber unerwarteten Ereignissen in den einzelnen Szenarien analysiert werden.

\subsection{Szenarienkonstruktion und Auswahl der Szenarien}

Die ausgewählten und im ersten Szenario-Workshop diskutierten Bausteine aus den Bereichen Klima, Wirtschaft, Soziales, Politik und Umwelt bildeten die Basis für die Szenarienkonstruktion. Für jeden Baustein (z. B. Bevölkerungsstruktur und Bevölkerungsentwicklung) wurden zwei bis vier unterschiedliche Entwicklungsmöglichkeiten festgelegt, die jeweils unterschiedliche Konsequenzen für die Gesamtentwicklung nach sich ziehen. Für die Szenarienkonstruktion wurden zudem der Einfluss der Klimaänderungen auf die Bausteine und Wechselwirkungen zwischen den Bausteinen integriert.

Die Entwicklungsmöglichkeiten der einzelnen Bausteine (vgl. Kap. 3) wurden in Verbindung mit unterschiedlich starken Klimaänderungen in verschiedenen Szenarien zusammengefasst, die jeweils ein in sich schlüssiges und plausibles und damit konsistentes Bild einer möglichen Zukunft abbilden (vgl. Abb. 3). Somit wurden alle Bausteine in jedem der vorgestellten Szenarien berücksichtigt. Aufgrund der Vielzahl der Bausteine und deren Entwicklungspfaden wurde als Hilfsmittel das Programm Szenoplan verwendet. Hier erfolgte eine Konsistenzanalyse der theoretisch möglichen Bausteinkombinationen zu Szenarien. Dabei wurden alle Entwicklungspfade jedes Bausteins mit allen anderen Entwicklungspfaden der anderen Bausteine in Verbindung gesetzt. Aus diesen wurden durch die Forschergruppe sechs Rohszenarien ausgewählt.

Nach der Diskussion der sechs Rohszenarien mit der Kerngruppe wurden vier Szenarien bestimmt, die den Auswahlkriterien Unterscheidbarkeit, Konsistenz, Interpretierbarkeit und Abbildung einer möglichst großen Bandbreite alternativer Zukünfte Rechnung tragen:

- Szenario 1: „Entwicklung in Grenzen“

- Szenario 2: „Alles in Maßen“

- Szenario 3: „Klima der Extreme“

- Szenario 4: „Divers geprägt“
Abb. 3 Der Prozess zur Erstellung der Szenarien. (Grafik: Meike Albers)

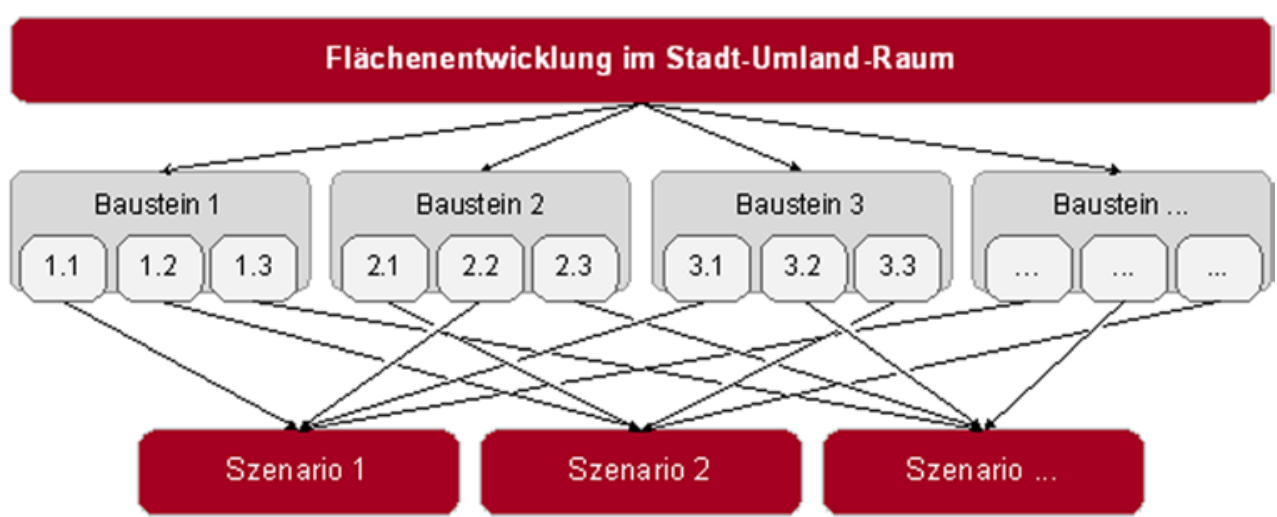


Tab. 1 Gegenüberstellung der vier Szenarien zur Flächenentwicklung im Stadt-Umland-Raum Rostock bis 2050

\begin{tabular}{|c|c|c|c|c|}
\hline Szenario & Entwicklung in Grenzen & Alles in Maßen & Klima der Extreme & Divers geprägt \\
\hline \multirow[t]{6}{*}{ Klimaänderungen } & $\begin{array}{l}\text { Mäßig starke } \\
\text { Klimaänderungen }\end{array}$ & Mäßige Klimaänderungen & Starke Klimaänderungen & $\begin{array}{l}\text { Unterschiedlich ausge- } \\
\text { prägte Klimaänderungen }\end{array}$ \\
\hline & $60 \mathrm{~cm}$ & $20 \mathrm{~cm}$ & $100 \mathrm{~cm}$ & $60 \mathrm{~cm}$ \\
\hline & Meeresspiegelanstieg & Meeresspiegelanstieg & Meeresspiegelanstieg & Meeresspiegelanstieg \\
\hline & $\begin{array}{l}1,6^{\circ} \mathrm{C} \\
\text { Temperaturerhöhung }\end{array}$ & $1{ }^{\circ} \mathrm{C}$ Temperaturerhöhung & $\begin{array}{l}2,2^{\circ} \mathrm{C} \\
\text { Temperaturerhöhung }\end{array}$ & $\begin{array}{l}2,2^{\circ} \mathrm{C} \\
\text { Temperaturerhöhung }\end{array}$ \\
\hline & $\begin{array}{l}\text { Zunahme } \\
\text { Winterniederschläge }\end{array}$ & $\begin{array}{l}\text { Geringe Zunahme } \\
\text { Winterniederschläge }\end{array}$ & $\begin{array}{l}\text { Starke Zunahme } \\
\text { Winterniederschläge }\end{array}$ & $\begin{array}{l}\text { Geringe Zunahme } \\
\text { Winterniederschläge }\end{array}$ \\
\hline & $\begin{array}{l}\text { Mittlerer Anstieg von } \\
\text { Extremwetterereignissen }\end{array}$ & $\begin{array}{l}\text { Geringer Anstieg von } \\
\text { Extremwetterereignissen }\end{array}$ & $\begin{array}{l}\text { Starker Anstieg von } \\
\text { Extremwetterereignissen }\end{array}$ & $\begin{array}{l}\text { Starker Anstieg von } \\
\text { Extremwetterereignissen }\end{array}$ \\
\hline $\begin{array}{l}\text { Soziale/gesellschaftliche } \\
\text { Aspekte }\end{array}$ & $\begin{array}{l}\text { Bevölkerungsrückgang } \\
\text { in Rostock, leichter Zu- } \\
\text { wachs im Umland mit } \\
\text { Siedlungsentwicklung, } \\
\text { auch in Konfliktbereichen }\end{array}$ & Bevölkerungszuwachs & $\begin{array}{l}\text { Bevölkerungsrückgang } \\
\text { in Rostock, leichter Zu- } \\
\text { wachs im Umland mit } \\
\text { Siedlungsentwicklung, } \\
\text { auch in Konfliktbereichen }\end{array}$ & $\begin{array}{l}\text { Positive Bevölkerungs- } \\
\text { entwicklung in Rostock } \\
\text { in Verbindung mit } \\
\text { Reurbanisierung }\end{array}$ \\
\hline \multirow[t]{4}{*}{ Ökonomische Aspekte } & $\begin{array}{l}\text { Positive Hafen- und Som- } \\
\text { mertourismusentwicklung }\end{array}$ & $\begin{array}{l}\text { Positive Hafen- und Som- } \\
\text { mertourismusentwicklung }\end{array}$ & $\begin{array}{l}\text { Positive Sommertouris- } \\
\text { musentwicklung }\end{array}$ & $\begin{array}{l}\text { Diversifizierung des } \\
\text { Tourismus }\end{array}$ \\
\hline & $\begin{array}{l}\text { Ausbau der } \\
\text { Verkehrsinfrastruktur }\end{array}$ & $\begin{array}{l}\text { Ausbau der } \\
\text { Verkehrsinfrastruktur }\end{array}$ & $\begin{array}{l}\text { Bedeutungsrückgang des } \\
\text { Hafens }\end{array}$ & $\begin{array}{l}\text { Hafenentwicklung auf be- } \\
\text { stehenden Flächen }\end{array}$ \\
\hline & $\begin{array}{l}\text { Intensivierung der Land- } \\
\text { wirtschaft zur Erzeugung } \\
\text { regenerativer Energien }\end{array}$ & $\begin{array}{l}\text { Intensivierung der Land- } \\
\text { wirtschaft zur Erzeugung } \\
\text { regenerativer Energien }\end{array}$ & $\begin{array}{l}\text { Erschließung neuer tou- } \\
\text { ristischer Zentren }\end{array}$ & $\begin{array}{l}\text { Regionales } \\
\text { Mobilitätskonzept }\end{array}$ \\
\hline & & & $\begin{array}{l}\text { Extensivierung der } \\
\text { Landwirtschaft und } \\
\text { Biolandbau }\end{array}$ & \\
\hline \multirow[t]{2}{*}{ Ökologische Aspekte } & $\begin{array}{l}\text { Zunahme der } \\
\text { Landschaftszerschneidung }\end{array}$ & $\begin{array}{l}\text { Starke Abnahme der } \\
\text { Freiflächen }\end{array}$ & $\begin{array}{l}\text { Starke Abnahme der } \\
\text { Freiflächen }\end{array}$ & Erhalt von Freiräumen \\
\hline & $\begin{array}{l}\text { Rückgang der } \\
\text { Wasserqualität }\end{array}$ & $\begin{array}{l}\text { Rückgang der Wasser- } \\
\text { qualität und erhöhter } \\
\text { Oberflächenabfluss, } \\
\text { starke Versiegelung }\end{array}$ & $\begin{array}{l}\text { Rückgang der Wasser- } \\
\text { qualität und erhöhter } \\
\text { Oberflächenabfluss, } \\
\text { starke Versiegelung }\end{array}$ & $\begin{array}{l}\text { Rückgang der } \\
\text { Wasserqualität }\end{array}$ \\
\hline \multirow[t]{3}{*}{ Politische Aspekte } & $\begin{array}{l}\text { Fokus auf } \\
\text { Wirtschaftsentwicklung }\end{array}$ & $\begin{array}{l}\text { Verbesserung der } \\
\text { Finanzsituation }\end{array}$ & $\begin{array}{l}\text { Fokus auf } \\
\text { Wirtschaftsentwicklung }\end{array}$ & $\begin{array}{l}\text { Fokus auf nach- } \\
\text { haltige Stadt- und } \\
\text { Regionalentwicklung }\end{array}$ \\
\hline & $\begin{array}{l}\text { Angespannte } \\
\text { Finanzsituation }\end{array}$ & $\begin{array}{l}\text { Stärkung der Stadt-Um- } \\
\text { land-Kooperation }\end{array}$ & $\begin{array}{l}\text { Angespannte } \\
\text { Finanzsituation }\end{array}$ & $\begin{array}{l}\text { Stärkung der Stadt-Um- } \\
\text { land-Kooperation }\end{array}$ \\
\hline & $\begin{array}{l}\text { Konkurrenz zwischen } \\
\text { Stadt und Umland }\end{array}$ & & $\begin{array}{l}\text { Konkurrenz zwischen } \\
\text { Stadt und Umland }\end{array}$ & \\
\hline
\end{tabular}

\section{2 Überblick über die Szenarien}

Die vier Szenarien unterscheiden sich stark in den integrierten Entwicklungspfaden der einzelnen Bausteine und deren Kombination (vgl. Tab. 1). Bezüglich der Klimaänderungen wurden aufgrund des Rahmenthemas „Anpassung an die Folgen des Klimawandels" in den Szenarien unterschiedlich starke Klimaänderungen angenommen, so dass jeweils ein Szenario geringe, mittlere und starke Klimaänderungen repräsentiert und ein Szenario unterschiedlich intensive Klimaänderungen beinhaltet, etwa starke Zunahme der Extremwetterereignisse und mittlerer Anstieg $(60 \mathrm{~cm})$ des Meeresspiegels. Bei den gesellschaftlichen Handlungsfeldern wurde in der Szenarienauswahl darauf geachtet, dass möglichst viele unterschiedliche
Entwicklungspfade der Bausteine in den Szenarien repräsentiert werden und dabei dennoch eine möglichst große Konsistenz des Gesamtszenarios erhalten bleibt, die Entwicklungen also plausibel aufeinander aufbauen. Die Szenarien skizzieren Bilder der Zukunft und veranschaulichen mögliche Situationen im Jahr 2050. Die darin enthaltenen Entwicklungspfade gehen von einem Zeithorizont von heute bis zum Jahr 2050 aus.

\subsubsection{Szenario 1: „Entwicklung in Grenzen“}

Im Szenario 1 „Entwicklung in Grenzen“ (vgl. Abb. 4) wirkt sich der Klimawandel bis zum Jahr 2050 durch einen Meeresspiegelanstieg von $60 \mathrm{~cm}$ und eine Temperaturerhöhung von $1,6^{\circ} \mathrm{C}$ aus. Die Niederschläge im Winter haben zuge- 
Abb. 4 Szenario 1 ,Entwicklung in Grenzen“. (Grafik: Paul Langfermann)
Abb. 5 Szenario 2 ,Alles in Maßen“. (Grafik: Paul Langfermann)
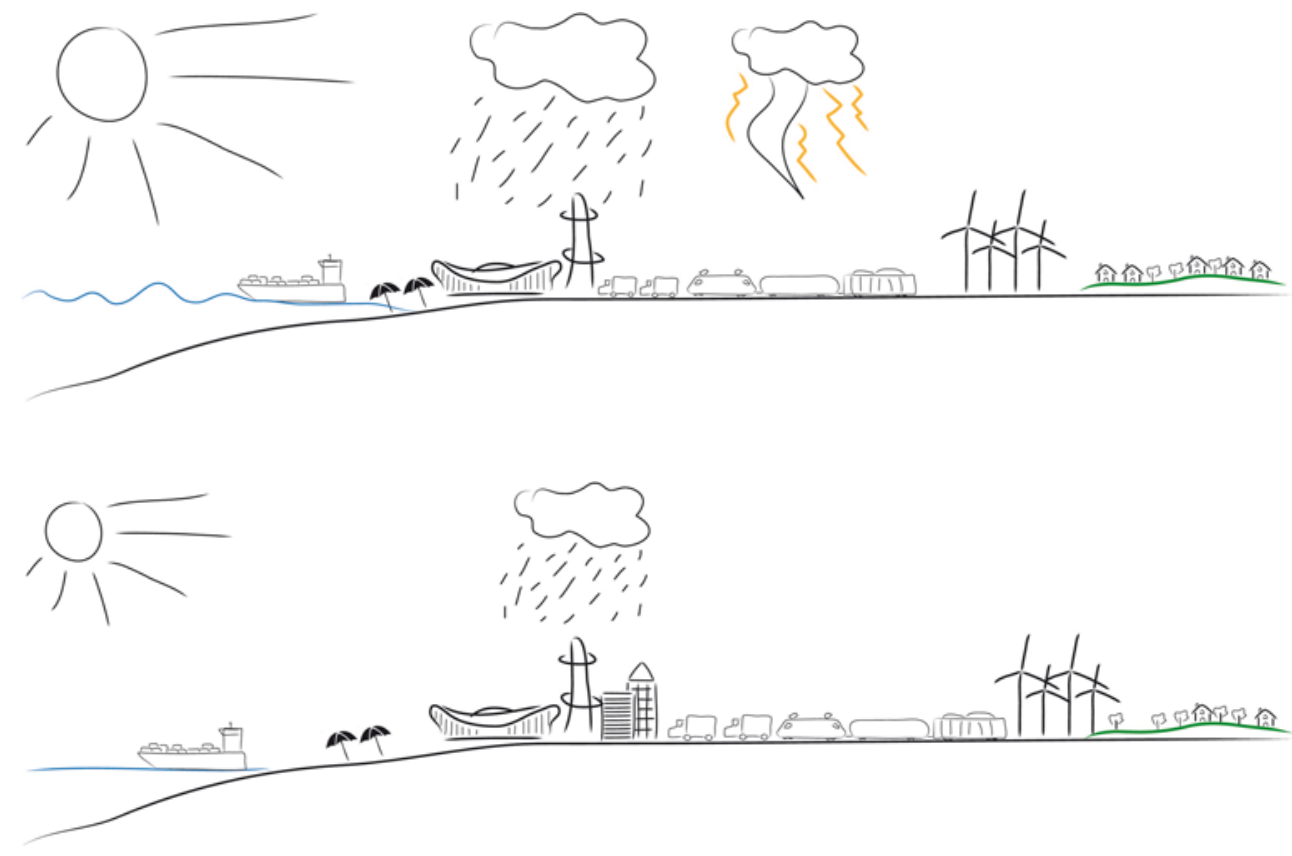

Abb. 6 Szenario 3 ,Klima der Extreme“. (Grafik: Paul Langfermann)

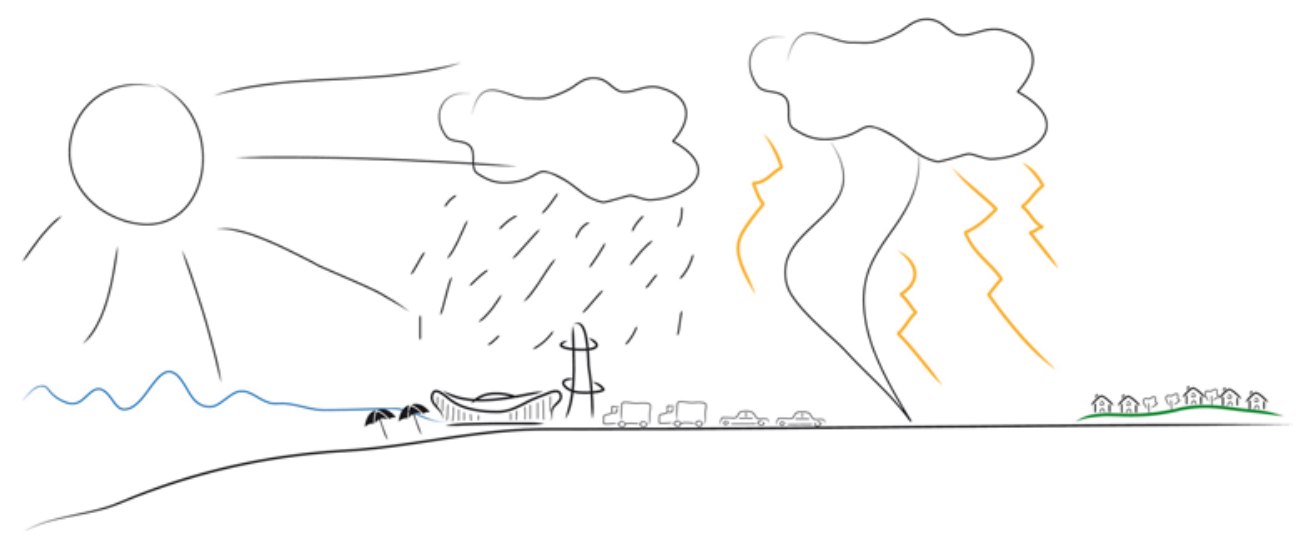

nommen und es gibt spürbar mehr Extremwetterereignisse. Hohe Kosten haben den Fokus auf die wirtschaftliche Entwicklung gelenkt, wozu unter anderem auch die Verkehrsinfrastruktur, insbesondere Straßen, ausgebaut wurde. Der Hafen und der Sommertourismus haben sich in diesem Szenario positiv entwickelt. Durch die Wirtschafts- und Siedlungsentwicklung hat die Landschaftszerschneidung im Umland zugenommen. Im Energiebereich liegt der Fokus auf regenerativen Energien, einhergehend mit einer Intensivierung der Landwirtschaft und einem Rückgang der Wasserqualität.

\subsubsection{Szenario 2: „Alles in Maßen“}

Das zweite Szenario „Alles in Maßen“(vgl. Abb. 5) geht von geringen Auswirkungen des Klimawandels aus - Anstieg des Meeresspiegels um $20 \mathrm{~cm}$, Temperaturerhöhung um $1{ }^{\circ} \mathrm{C}$ und geringer Anstieg von Extremwetterereignissen.
Auch die klimatisch günstigen Bedingungen haben zu einer positiven Bevölkerungsentwicklung und zu einem Ausbau des Sommertourismus beigetragen. Der Hafen und die maritime Wirtschaft konnten sich ebenfalls positiv entwickeln. Die Folge dieser Entwicklungen ist eine starke Verringerung von Freiflächen und eine Verschlechterung der Wasserqualität sowie stärkerer Oberflächenabfluss, etwa durch zunehmende Versiegelung.

\subsubsection{Szenario 3: „Klima der Extreme“}

Im Szenario „Klima der Extreme“ (vgl. Abb. 6) entfaltet der Klimawandel erhebliche Auswirkungen. Der Meeresspiegel ist bis zum Jahr 2050 um einen Meter angestiegen und die Durchschnittstemperatur hat sich um $2,2^{\circ} \mathrm{C}$ erhöht. Insbesondere im Winter gibt es deutlich mehr Niederschläge. Extremwetterereignisse wie Stürme, Starkregen oder Hitzewellen treten deutlich häufiger auf. Diese Klimaän- 
Abb. 7 Szenario 4 ,Divers geprägt". (Grafik: Paul Langfermann)

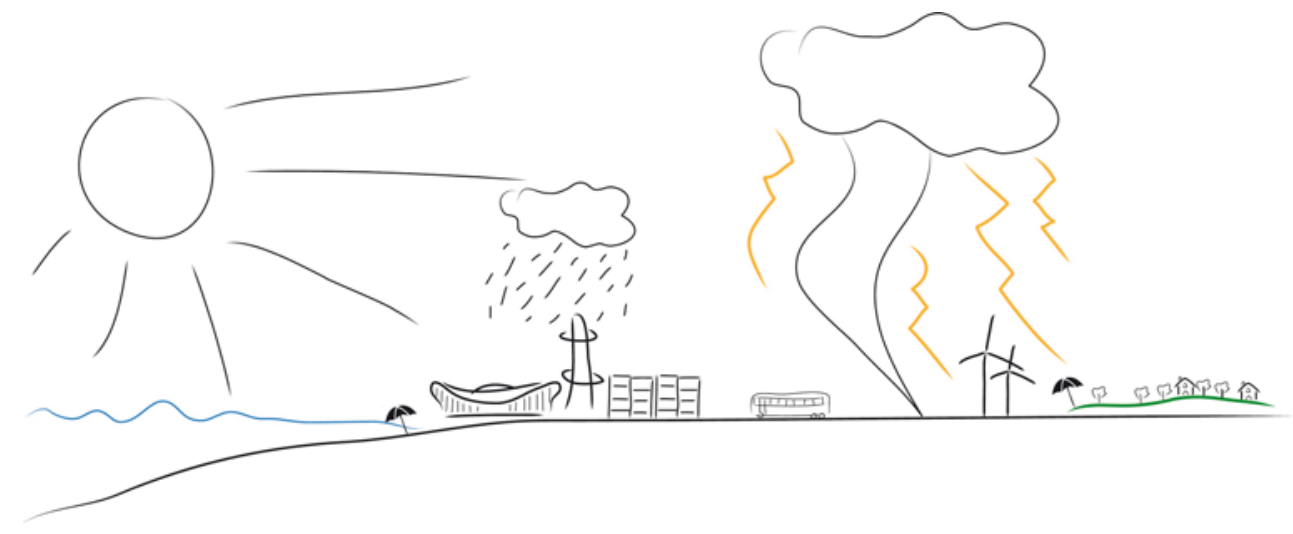

derungen werden von Suburbanisierung und einer Konkurrenzsituation zwischen Stadt und Umland begleitet. Durch die Siedlungsentwicklung im Umland und den Ausbau des Tourismus kommt es zu einem Verlust von Freiflächen und zu einer Nutzung von überschwemmungsgefährdeten Gebieten. Die Landwirtschaft setzt eher auf Extensivierung durch weniger anfälligen Biolandbau. Eine Fokussierung auf regionale Produkte in Verbindung mit der Häufung von Extremwetterereignissen führt zu einem Bedeutungsverlust für den Hafen. Regenerative Energien nehmen zu, werden jedoch nicht in der Region produziert, sondern vorwiegend zugekauft.

\subsubsection{Szenario 4: „Divers geprägt“}

Im Szenario „Divers geprägt“ (vgl. Abb. 7) äußert sich der Klimawandel in unterschiedlichen Intensitäten. Während der Meeresspiegel bis zum Jahr 2050 um $60 \mathrm{~cm}$ angestiegen ist, hat sich die Temperatur um $2,2^{\circ} \mathrm{C}$ stark erhöht und Extremwetterereignisse treten sehr viel häufiger auf. Insbesondere im Winter lassen sich mehr Niederschläge feststellen. Der Stadt-Umland-Raum Rostock hat den Fokus auf eine nachhaltige Entwicklung gelegt, wozu auch der Erhalt von Freiräumen zählt. Bevölkerungswachstum findet in Rostock statt, während die Bevölkerungszahl im Umland stagniert. Der Hafen und die maritime Wirtschaft entwickeln sich auf den bereits bestehenden Flächen. Insgesamt herrscht ein hohes Bewusstsein in der Region für das Thema Klimawandel und sorgt z. B. auch für den Ausbau des Wintertourismus. Ein regionales Mobilitätskonzept wird konsequent umgesetzt, um Verlagerungen vom Wasser auf Straße und Schiene aufzufangen.

\section{Zusammenfassung und Ausblick}

In diesem Beitrag wurde die inhaltliche Erarbeitung der Szenarien für die Flächenentwicklung im Stadt-UmlandRaum Rostock vorgestellt. Die gemeinsam mit den lokalen und regionalen Akteuren entwickelten Szenarien zeigen vier unterschiedliche Bilder, wie sich der Stadt-Umland-Raum Rostock bis zum Jahr 2050 entwickeln könnte. Die Szenarien beinhalten unterschiedliche Entwicklungsmöglichkeiten aus insgesamt 18 Bausteinen, die nach Einschätzung der Akteure von zentraler Bedeutung für die Flächenentwicklung in der Region sind. Dabei wurden sowohl die Auswirkungen des Klimawandels als auch weitere ökologische und gesellschaftliche Aspekte berücksichtigt, da auch deren Entwicklung - ebenso wie die des Klimas - derzeit nicht vorhergesehen werden kann. Insgesamt konnten so mit den Szenarien vier mögliche Entwicklungen abgebildet werden, die zum einen der Unsicherheit, zum anderen der Komplexität künftiger Entwicklungen gerecht werden.

Die Auseinandersetzung mit den zahlreichen Bausteinen und deren komplexen Zusammenhängen stellt eine Besonderheit des Szenario-Planungs-Prozesses in Rostock dar, die auch von den Akteuren positiv bewertet wurde. Nach deren Aussagen findet eine derart komplexe Auseinandersetzung mit künftigen Entwicklungen in der Planungspraxis üblicherweise nicht statt. Hier wurde durch den Szenario-PlanungsProzess ein eindeutiger Mehrwert gegenüber den in der Praxis üblichen Vorgehensweisen, aber auch anderen Methoden, die einen sektoralen Ansatz verfolgen, erzielt. Ermöglicht wurde die Integration der Komplexität durch die aufeinander aufbauenden Workshops: So fand zunächst eine Komplexitätsreduktion statt, indem im ersten Workshop die einzelnen Bausteine mit ihren jeweiligen Entwicklungsmöglichkeiten diskutiert wurden. Im zweiten Workshop wurden die Bausteine dann zu vier Szenarien gebündelt und die Wechselwirkungen zwischen den Bausteinen diskutiert. Die einzelnen Szenarien ermöglichten dann eine integrierte Betrachtung aller Bausteine und damit eines komplexen Bildes.

Die Erarbeitung von vier unterschiedlichen Szenarien ermöglichte es, sich mit der Unsicherheit auseinanderzusetzen, die sowohl dem Klimawandel als auch den anderen zukünftigen gesellschaftlichen Entwicklungen innewohnt. Obwohl die Auseinandersetzung mit künftigen und damit unsicheren Entwicklungen für die Planung kein grundsätzlich neues Thema darstellt, wird die Unsicherheit des Klimawandels als besondere Herausforderung angesehen. Anhand 
der Bandbreite der Szenarien konnten ein breiter Maßnahmenkatalog und Strategien erarbeitet und bewertet und im Hinblick auf ihre Zukunftsfähigkeit beurteilt werden. Durch die aufbauend auf den Szenarien erarbeiteten Strategien und konkreten Maßnahmen hat der Stadt-Umland-Raum Rostock Ansatzpunkte für eine praktische Implementierung von Maßnahmen zur Anpassung an die Folgen des Klimawandels. Basierend auf den vier Szenarien kann auch im Anschluss an den Szenario-Planungs-Prozess im StadtUmland-Raum Rostock eine Diskussion über mögliche Strategien und Maßnahmen erfolgen, die nicht nur einer klar definierten, sondern möglicherweise auch unterschiedlichen Entwicklungen gerecht wird.

Darüber hinaus haben sich die relevanten lokalen und regionalen Akteure im Rahmen des Szenario-PlanungsProzesses mit den möglichen künftigen Entwicklungen auseinandergesetzt und einen Dialog begonnen, der für die Entwicklung und insbesondere für die Umsetzung möglicher Strategien und Maßnahmen zur Anpassung an die Folgen des Klimawandels von besonderer Bedeutung ist.

Förderhinweis Die Forschungsnachwuchsgruppe „plan B:altic“ wird vom Bundesministerium für Bildung und Forschung innerhalb des Rahmenprogramms „FONA - Forschung für nachhaltige Entwicklungen“ im sozial-ökologischen Förderschwerpunkt von November 2009 bis Juni 2014 unter dem Förderkennzeichen 01UU0909 gefördert.

\section{Literatur}

Allison, I.; Bindoff, N. L.; Bindschadler, R. A.; Cox, P. M.; de Noblet, N.; England, M. H.; Francis, J. E.; Gruber, N.; Haywood, A. M.; Karoly, D. J.; Kaser, G.; Le Quéré, C.; Lenton, T. M.; Mann, M. E.; McNeil, B. I.; Pitman, A. J.; Rahmstorf, S.; Rignot, E.; Schellnhuber, H. J.; Schneider, S. H.; Sherwood, S. C.; Somerville, R. C. J.; Steffen, K.; Steig, E. J.; Visbeck, M.; Weaver, A. J. (2009): The Copenhagen Diagnosis. Updating the world on the latest climate science. Sydney.

Amt für Raumordnung und Landesplanung Mittleres Mecklenburg/ Rostock (2011): Entwicklungsrahmen Stadt-Umland-Raum Rostock. Rostock. Online unter: http://www.rpv-mmr.de/fileadmin/ pdf/Stadt-Umland-Planung/entwicklungsrahmen_2011/SUR_ ER_August_2011_korrekt.pdf (letzter Zugriff am 27.06.2013).

BALTEX Assessment of Climate Change for the Baltic Sea Basin (2008): Assessment of climate change for the Baltic Sea Basin. Berlin, Heidelberg.

Bärring, L.; von Storch, H. (2004): Scandinavian storminess since about 1800. In: Geophysical Research Letters 31 (20), doi: 10.1029/2004GL020441.

Bundesministerium für Verkehr, Bau und Stadtentwicklung; Bundesinstitut für Bau-, Stadt- und Raumforschung (2009): Klimawandelgerechte Stadtentwicklung. Rolle der bestehenden städtebaulichen Leitbilder und Instrumente. Bonn. = BBSR-Online-Publikation 24/2009. Online unter: http://www.bbsr.bund. de/cln_032/nn_21272/BBSR/DE/Veroeffentlichungen/BBSROnline/2009/DL_ON242009, templateId=raw,property=publicationFile.pdf/DL_ON242009.pdf (letzter Zugriff am 27.06.2013).

Böschen, S.; Weis, K. (2007): Die Gegenwart der Zukunft: Perspektiven zeitkritischer Wissenspolitik. Wiesbaden.
Buttschardt, T. (2010): Creeping Disasters als Folge schleichender Umweltveränderungen? - Ein Konzeptvorschlag. In: Forum Geoökologie 21 (2), 16-27.

Daschkeit, A.; Sterr, H. (2005): Klimawandel und Küstenschutz. Hat Sylt eine Zukunft? In: Glaeser, B. (Hrsg.): Küste, Ökologie und Mensch. Integriertes Küstenmanagement als Instrument nachhaltiger Entwicklung. München, 267-292.

Davoudi, S. (2009): Framing the role of spatial planning in climate change. = Electronic Working Paper No. 43. Online unter: http:// www.ncl.ac.uk/guru/publications/working/documents/EWP43. pdf (letzter Zugriff am 27.06.2013).

Fink, A.; Siebe, A. (2006): Handbuch Zukunftsmanagement. Frankfurt am Main.

Fleischhauer, M.; Bornefeld, B. (2006): Klimawandel und Raumplanung. Ansatzpunkte der Raumordnung und Bauleitplanung für den Klimaschutz und die Anpassung an den Klimawandel. In: Raumforschung und Raumordnung 64 (3), 161-171.

Greiving, S.; Fleischhauer, M. (2008): Raumplanung: in Zeiten des Klimawandels wichtiger denn je! Größere Planungsflexibilität durch informelle Ansätze einer Klimarisiko-Governance. In: Raumplanung 137, 61-66.

Hallegatte, S. (2009): Strategies to adapt to an uncertain climate change. In: Global Environmental Change 19 (2), 240-247.

Heiland, S.; Kowarik, I. (2008): Anpassungserfordernisse des Naturschutzes und seiner Instrumente an den Klimawandel und dessen Folgewirkungen. In: Informationen zur Raumentwicklung 6/7, 415-422.

Helmholtz-Zentrum Geesthacht (2011): Norddeutscher Klimaatlas. Geesthacht. Online unter: http://www.norddeutscher-klimaatlas. de/ (letzter Zugriff am 26.06.2013).

Intergovernmental Panel on Climate Change (2007): Climate Change 2007: Synthesis Report. Paris.

Kahn, H.; Wiener, A. J. (1967): The year 2000. New York.

Meier, H. E. M.; Bromann, B.; Kjellström, E. (2004): Simulated sea level in past and future climates of the Baltic Sea. In: Climate Research 27 (1), 59-75.

Mietzner, D. (2009): Strategische Vorausschau und Szenarioanalysen. Methodenevaluation und neue Ansätze. Wiesbaden.

National Oceanic and Atmospheric Administration (2012): Mean Sea Level Trend. 120-012 Warnemunde, Germany. Online unter: http://co-ops.nos.noaa.gov/sltrends/sltrends_global_station. shtml?stnid=120-012 (letzter Zugriff am 22.07.2013).

O'Brian, K. (2010): Responding to environmental change: A new age for human geography? In: Progress in Human Geography 35 (4), $542-549$.

Overbeck, G.; Hartz, A.; Fleischhauer, M. (2008): Ein 10-Punkte-Plan „Klimaanpassung“. Raumentwicklungsstrategien zum Klimawandel im Überblick. In: Informationen zur Raumentwicklung 6/7, 363-380.

Peterson, G. D.; Cumming, G. S.; Carpenter, S. R. (2003): Scenario Planning: A Tool for Conservation in an Uncertain World. In: Conservation Biology 17 (2), 358-366.

Ritter, E.-H. (2007): Klimawandel - Eine Herausforderung an die Raumplanung. In Raumforschung und Raumordnung 65 (6), 531-538.

Scholles, F. (2008): Szenariotechnik. In: Fürst, D.; Scholles, F. (Hrsg.): Handbuch Theorien und Methoden der Raum- und Umweltplanung. Dortmund, 380-392.

Stehr, N.; von Storch, H. (2008): Anpassung und Vermeidung oder von der Illusion der Differenz. In: GAIA 17 (3), 270-273.

Stiens, G. (1998): Prognosen und Szenarien in der räumlichen Planung. In: Akademie für Raumforschung und Landesplanung (Hrsg.): Methoden und Instrumente räumlicher Planung. Hannover, 113-145.

von Reibnitz, U. (1992): Szenariotechnik. Instrumente für die unternehmerische und persönliche Erfolgsplanung. Wiesbaden. 